\title{
Formal Analysis of a PoS Blockchain
}

\author{
Wai Yan Maung Maung Thin ${ }^{1}$, Naipeng Dong ${ }^{1}$, Guangdong Bai ${ }^{2}$, and Jin \\ Song Dong ${ }^{1}$ \\ 1 National University of Singapore \\ 2 Singapore Institute of Technology
}

\begin{abstract}
Blockchain technology relies on consensus algorithms to resolve conflicts in Byzantine environments. New blockchain algorithms are rapidly designed and implemented without a properly conducted formal analysis and verification. In this paper, we look at Tendermint which is a proof-of-stake consensus algorithm. We verified that the consensus protocol is deadlock-free and is able to reach consensus when at least $67 \%$ of the network is in agreement. We also proved that a minority set of nodes that compose more than $33 \%$ of the network is enough to censor the majority of the network and prevent the network from reaching consensus and conclude that the algorithm has some shortcomings when it comes to availability.
\end{abstract}

Keywords: formal verification, blockchain, tendermint, consensus, proofof-stake

\section{Introduction}

Introduced by Bitcoin in 2008 as a means to provide a trustworthy service among non-trusting peers without a central governing body, the blockchain technology has long evolved from its traditional use as a decentralized ledger for digital currencies. Many platforms such as Ethereum [5] and Ripple [11] are already using blockchains to support smart contracts which allow the execution of arbitrary codes in a decentralized manner [15]. The technology can also be easily applied to several fields such as Internet of Things (IoT) [16][9] or applications such as data privacy tools [17] and reputation systems [12].The growth of the ecosystem and the adoption of the blockchain technology in various domains and applications have created a competitive environment where newer protocols and algorithms are being rapidly introduced and implemented without a formal analysis and verification process. The lack of related published research work implies that many of the widely used protocols and algorithms have yet to undergo a formal analysis that verifies the security properties and the correctness of the system. This process is extremely important, especially for the consensus algorithms that mandate how to resolve conflicting claims in the absence of a central authority and define the consistency, performance, scalablity and liveliness of the system. Depending on the nature of the blockchain, the focus of the consensus algorithm is also different. For instance, Tendermint [8] favours consistency over liveliness 
(availability) whereas Casper [4], the consensus algorithm of Ethereum sacrifices consistency to achieve plausible liveness. Previous research works on formal analysis of blockchains [1] [7] [2] did not focus on the consensus protocols. The paper aims to fill the gap by providing a formal analysis of a blockchain using proof-of-stake as the consensus algorithm. We implement Tendermint consensus protocol in C\# and CSP\# [13] using the PAT [14] model checker. We prove that the consensus protocol is deadlock-free and is able to reach consensus when at least $67 \%$ of the network is in agreement. We also conclude that a minority set of nodes that compose more than $33 \%$ of the network can censor the majority of the network and prevent the network from reaching consensus and that the algorithm has some shortcomings when it comes to availability.

\section{Background}

\subsection{Blockchain Architecture}

A blockchain is a sequence of blocks, each of which maintains the hash value and the link to its previous block (with the exception of the genesis block which is the first block in the chain). This design architecture ensures that the contents for an existing block (for example, transactions) in the chain cannot be updated without modifying the hash values of the following blocks. Contents of a block widely vary based on the application. A typical block consists of a block header and a block body. The header is used to store the metadata of the parent and the current block, such as the signatures and the hash values. The body is used as a payload and can be used to store anything, ranging from messages and transactions to smart contracts.

A blockchain network is a set of nodes responsible for keeping track of the blockchain and validating and appending new blocks to the blockchain. Based on the difference in functionality, nodes can be categorized into two - miners and validators. Miners store only the last few blocks of the blockchain and are responsible for creating new blocks. Validators store a full copy of the blockchain and ensure that the new block submitted by the miners is valid and conforms to the rules of the blockchain. Based on the architecture of the blockchain, it is possible for a node to be both a miner and a validator at the same time.

\subsection{Consensus in Blockchains}

The nodes in the blockchain network operate under the fact that there is no central authority governing over them. In a perfect scenario, all the members of the network always agree on the same new block to be appended to the blockchain and there exists only a single blockchain in the whole network. In reality, however, nodes might get disconnected from the network or even act maliciously in Byzantine environments on purpose. Thus, a fault-tolerant consensus protocol is required which is agreed by all the nodes in the blockchain to resolve any potential conflict. 
Consensus protocols differ based on the aspects or the attributes of the node that are being assessed. Proof-of-work (PoW) requires the node to provide the computing power to solve a mathematical problem in order to append a new block to the blockchain. The most well-known user of PoW consensus is Bitcoin where miners have to solve cryptographic hash puzzles as proof of work. In Proofof-stake (PoS), a node has to stake something it owns, usually in the form of a cryptocurrency. If a malicious node try to manipulate the blockchain and the other nodes detect it, the locked-up stakes get slashed or rewards are withheld. Delegated proof-of-stake (DPoS) is where the nodes of the network vote for a set of nodes to be the delegators. Other less common protocols include proof-ofcorrectness (Ripple) and proof-of-burn (Slimcoin [10]).

In a PoW system, the nodes are rewarded for performing an operation that is agreed by a majority of the nodes in the system. The caveat here is that participants are not punished for performing a malicious operation. As a result, PoW systems cannot deter the participants from performing a selfish mining [6] or participating in a $51 \%$ attack. In order to solve this problem, newer generations of blockchains (Ethereum, Tendermint, etc.) have started to use proof-of-stake as the consensus algorithm. In a PoS system, the participants are rewarded for performing a non-malicious activity just like a PoW system but they are also held accountable and are punished for any malicious operation.

\subsection{Modeling Language}

Our Tendermint consenus model is built on top of CSP \# [13] and is verified using PAT [14] model checker. CSP\# integrates the high-level modeling operators of CSP with low-level procedural codes in C language. The following is a list of CSP\# syntax and semantics commonly used in modelling concurrent systems.

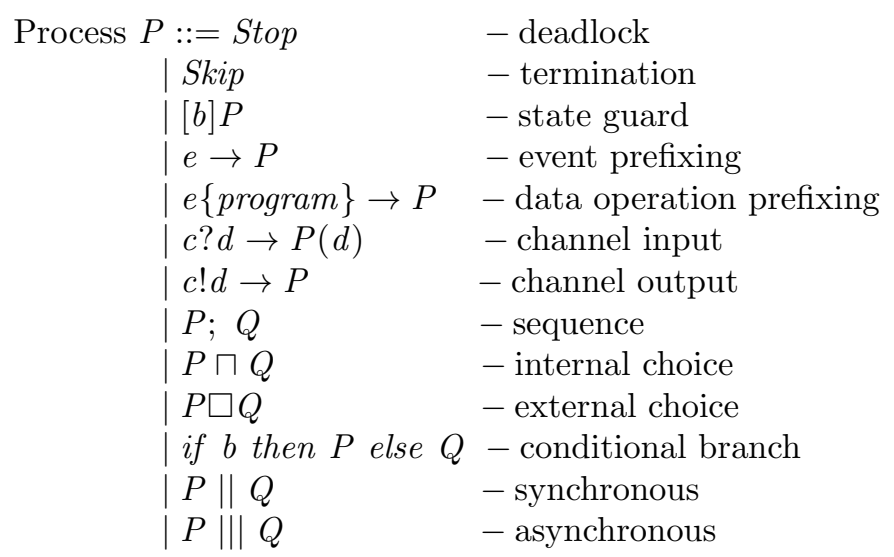

where $P$ and $Q$ are Processes, $b$ is a condition, $e$ is a simple event, program is a block of code that is atomically executed and $c$ is a synchronized communication channel.

CSP\# uses a Labeled Transition System (LTS) for model checking. This is represented as a tuple $\left(S, S_{\text {init }}, A, T\right)$ where $S$ is a finite set of states, $S_{\text {init }} \in S$ 
is the initial state, $A$ is a set of actions and $T$ is a set of labeled transition relations. $T$ is a set of tuples $\left(s, e, s^{\prime}\right)$ where event $e$ causes the system to change its state from $s$ to $s^{\prime}$. We say a state $s_{n}$ is reachable if there exists a path $P$ such that $\forall i<n ;\left(s_{i}, e_{i}, s_{i+1}^{\prime}\right) \in T \wedge s_{0}=S_{i n i t}$. We will be using this reachability checking to prove the security properties of the consensus algorithm.

\section{Tendermint}

Tendermint is a proof-of-stake consensus protocol that is Byzantine fault tolerant. Participants in the protocol are called validators. There is no concept of miners in Tendermint and thus, validators are also responsible for the creation of new blocks. The height of the chain increases every time a block is added to the chain. Validators are chosen in a round-robin manner to become the proposer who is in-charge of creating and proposing a block for the current round. Validators are required to post a bond transaction that will lock a set amount of his coins (stakes) for a set duration. If the validator is found to be involved in any malicious activity within this duration, it can be punished by slashing away its deposited stake. After this duration, the stakes will be unlocked and returned to the validator.

\subsection{Consensus Algorithm}

The consensus algorithm consists of five steps - Propose, Prevote, Precommit, Commit and NewHeight.

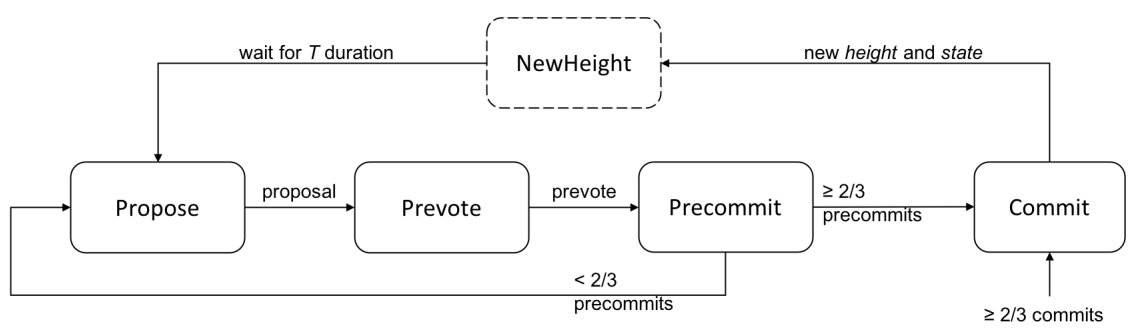

Fig. 1. State machine of the consensus protocol, adapted from Tendermint: Consensus without mining [8]

In Propose step, the proposer will broadcast a proposal to its peers. A proposal includes the block, the signatures of the validators who have validated the block, the signature of the proposer as well as the round and the height information. If the proposer has already locked on a block, that block will be used for proposal. Otherwise, a new block will be created. All nodes will gossip the proposal to their neighbouring peers during this period.

In Prevote step, each validator will vote for a block and gossip it to the neighbours. A vote consists of the hash of the voted block, the signature of the 
voter, type of vote - whether it is a prevote or a precommit plus the round and the height information. The block to be included is chosen in the following order - (1) a locked proposed block from prior rounds and (2) a valid acceptable block from the current proposal. If neither is available, a special NIL prevote is broadcast to the neighbours. All nodes will gossip all prevotes for the round to their neighbouring peers.

In Precommit step, the validator checks if it has received more than $2 / 3$ of prevotes for an acceptable block. If there is one, the validator releases the existing lock, locks onto this block and signs and broadcasts a precommit vote for this block. When a block is locked, the prevotes for the locked block are packaged into a proof-of-lock which will be used to create the block in the next Proposal. In the case where there are fewer than $2 / 3$ prevotes, the validator will neither sign nor lock on any block. During this period, all nodes will gossip all precommits for the round to all neighbouring peers. At the end of Precommit, if the node has received more than $2 / 3$ of precommits for a particular block, it will proceed to Commit step. Otherwise, it will transit to the Propose step of the next round.

In Commit step, two parallel conditions must be fulfilled before the consensus algorithm can cycle back to Propose step. First and foremost, the node is required to have the block committed by the network so that it can sign and broadcast a commit to the peers. Secondly, the node must wait until at least $2 / 3$ commits of the block are received by the network. Once these are satisfied, the node will set the CommitTime to current time and move on to NewHeight step where the nodes will stay for a fixed duration. The purpose is to allow the nodes to wait for additional commits of the committed block which were not received in Precommit due to network latency issues. After the set duration is up, the algorithm starts again from Propose. At anytime during the consensus process, if a node receives more than $2 / 3$ commits for a particular block, it will immediately enter the Commit step.

\section{$4 \quad$ Modelling}

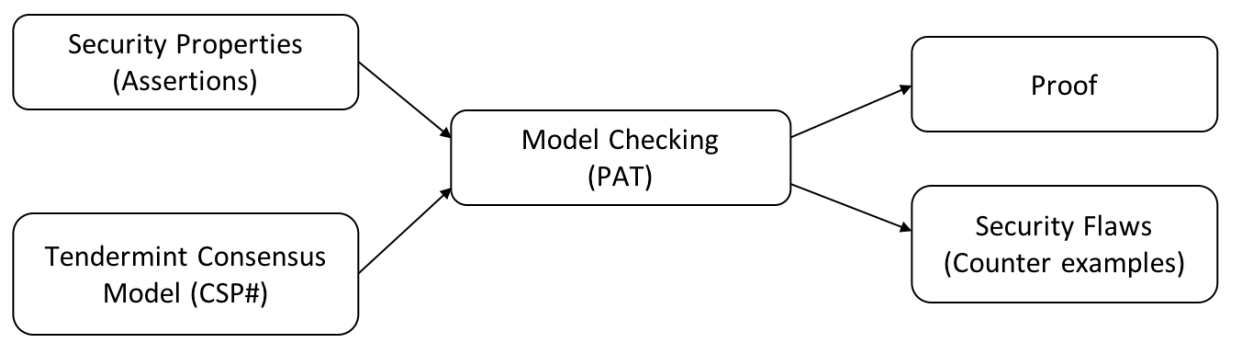

Fig. 2. Overview of the verification approach 
Figure 2 shows the overview of the verification approach. We first implement the Tendermint consensus model in CSP\# and define its security properties. We also define a set of assertion rules that we want to verify against the model. We run the simulation through PAT which gives us verification and the traces of the satisfied and unsatisfied assertions.

For the purpose of this paper, we will be focusing on the consensus protocol of the blockchain and as such we have abstracted the structure and the content of the block. We will be modelling the channel communication among nodes only for consensus. Data exchange among the nodes for the internal contents of the blocks such as transactions and contracts is considered to be outside of the scope of this work. We ran two sets of tests - one with 3 validators in the network and the other with 4 validators. The number of nodes in the network is limited to 4 to reduce the number of states in the system. This is enough to cover all the required test cases of the model where for 3 validators, 1 node means $33 \%$ of the network; 2 nodes means $67 \%$ and for 4 validators, 1 node means $<33 \%$; 2 nodes means between $33 \%$ and $67 \%$ and 3 nodes means $>67 \%$. The following assumptions have been made while designing the model.

1. All the nodes in the network are connected to each other.

2. Existing nodes will not leave the network and no new nodes will join the network.

3. All nodes have the same weight or voting power.

4. There is no latency in the network.

First, we define a set of variables used in the system.

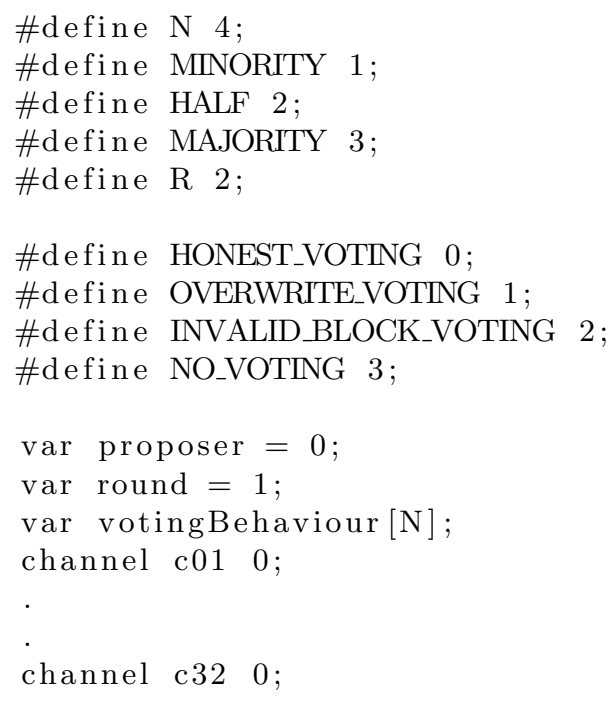

where $N$ is the number of nodes in the network and $R$ stands for the number of rounds to be simulated. proposer is the proposer chosen by round-robin for round. votingBehaviour is used to set the behavior of the nodes in the 
system which can be either one of the following values: HONEST_VOTING, OVERWRITE_VOTING, INVALID_BLOCK_VOTING, NO_VOTING. channel $c_{i j}$ represents a synchronized communication channel from node $\mathrm{i}$ to node $\mathrm{j}$.

\subsection{Blockchain}

We define a blockchain as a set of nodes $\mathrm{N}$ where each node $x \in N$ executes a sequence of processes in parallel. Since there is no network latency in our model, NewHeight is not required and Commit does not need to wait until the node receives the block. We also split the PreparePOL from Precommit since preparing proof-of-lock happens at the end of Precommit and not during. At the end of each round, NextRound() process is executed to select the next proposer.

BlockChain ()$=(|| x:\{0 \ldots N-1\} @(\operatorname{Propose}(\mathrm{x}) ; \operatorname{Prevote}(\mathrm{x}) ;$

Precommit (x); PreparePOL (x); Commit (x))); NextRound ();

\subsection{Propose}

The Propose() step is defined as follows. If a node is a proposer for this round, it will broadcast the proposal to other nodes using the communication channels. Otherwise, it will wait for a proposal from the proposer.

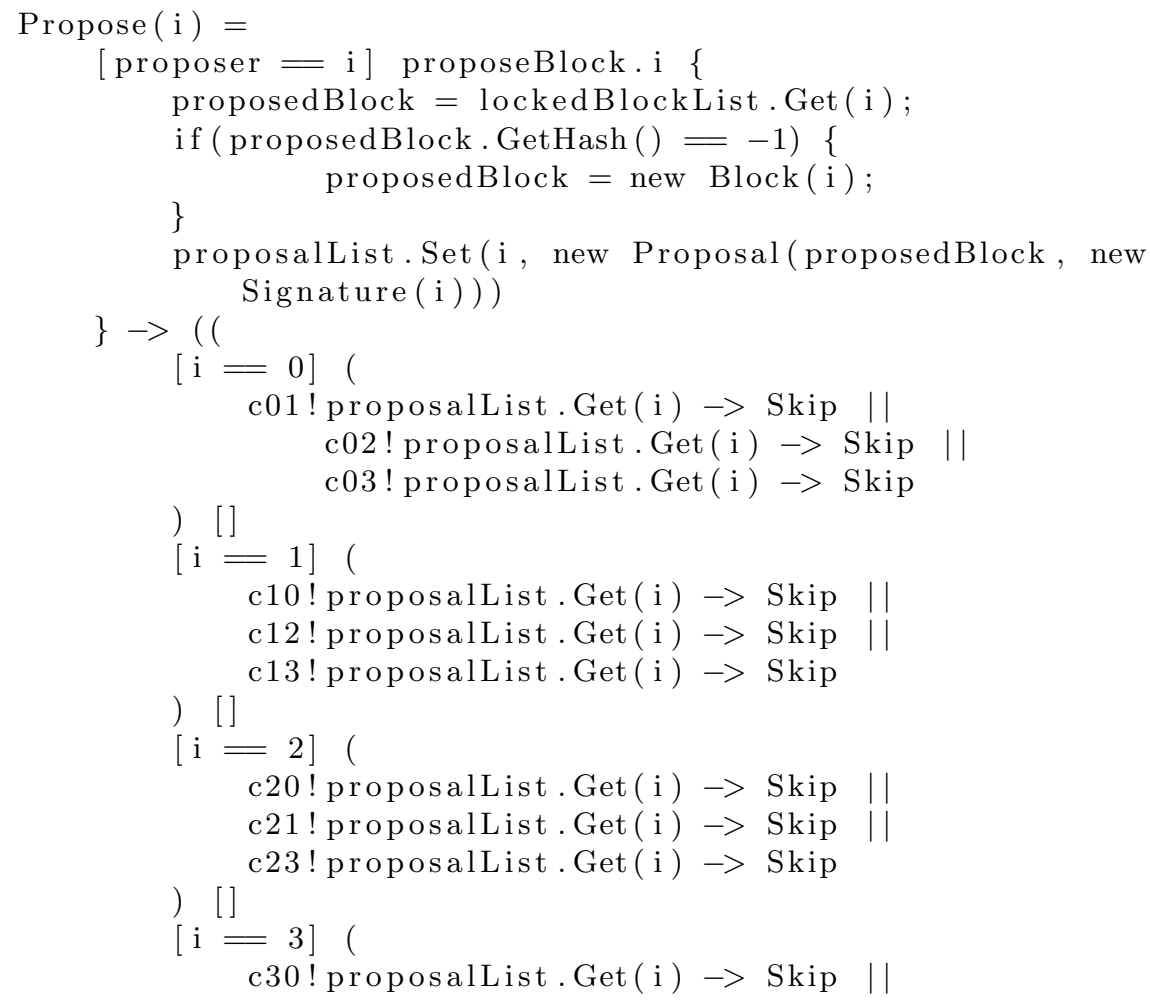




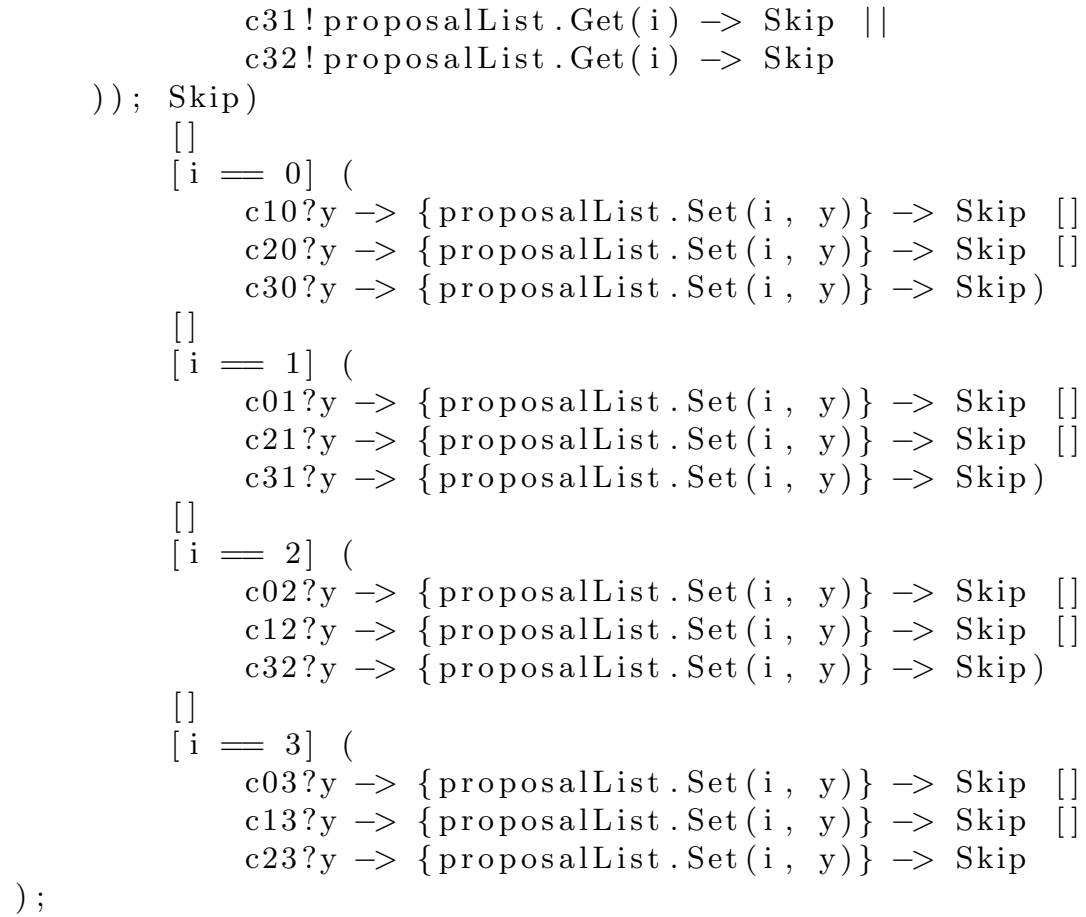

\subsection{Prevote}

The Prevote $(i)$ process is a sequence of two processes, prefixed by event propose_end which is used to represent the end of the Propose step. The first process Prevote_i() validates the proposal received from Propose $(i)$ and votes accordingly. The second process represents the broadcasting and the receiving of votes among the nodes. A node will not broadcast the vote if the censor flag is active. The definition of this process is slightly different for each node since different channels are used for communication. However, the logical behavior is the same for every node and has been marked with "..." below for readability.

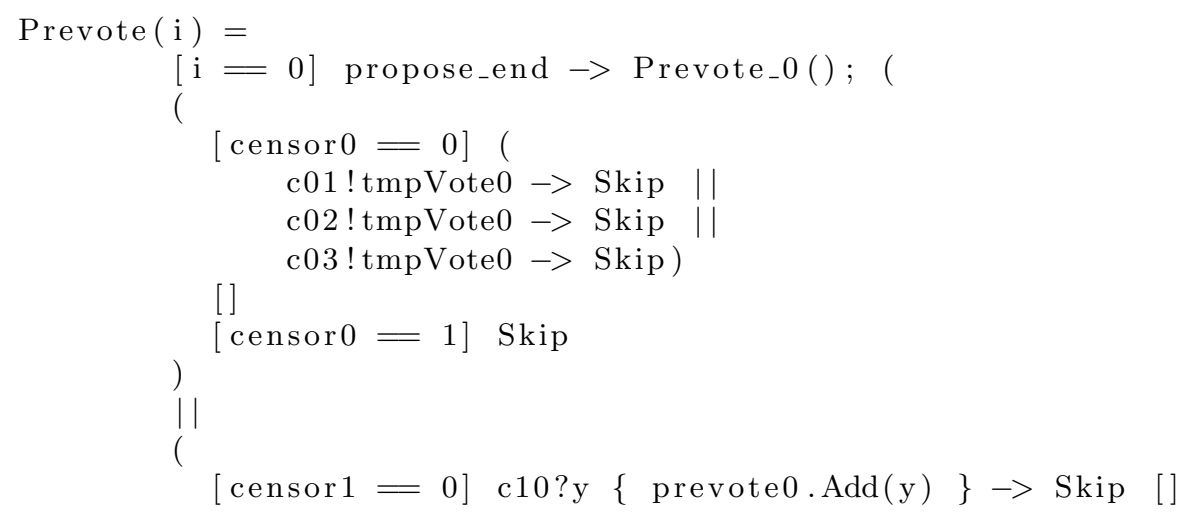




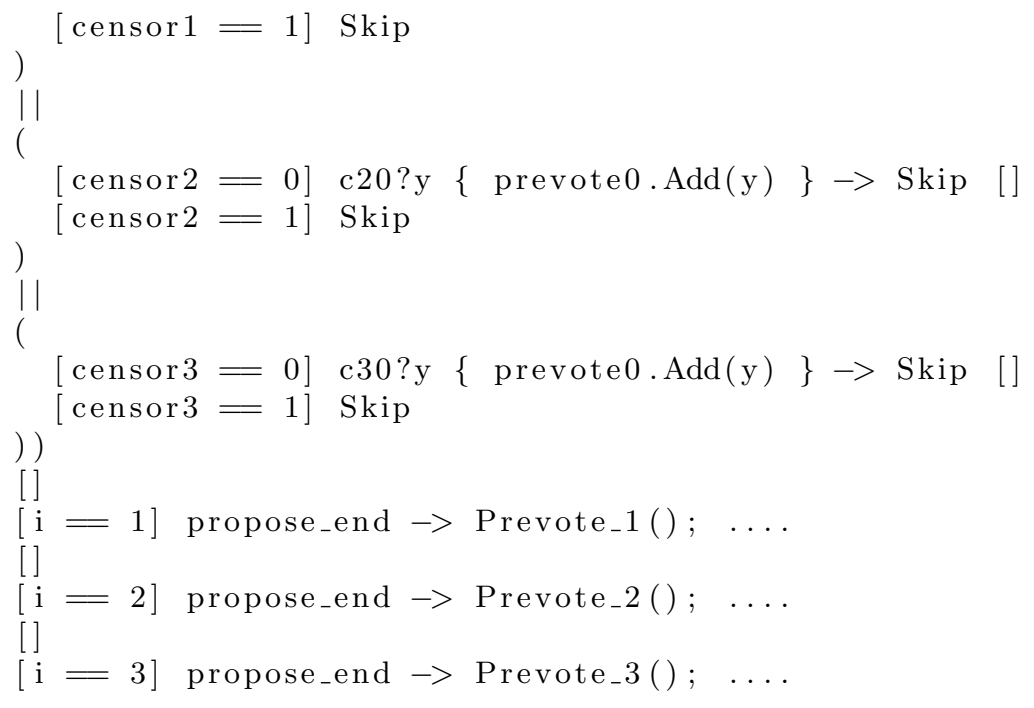

\subsection{Prevote}

Prevote_i() validates the proposal received during Propose and broadcasts a vote accordingly. The malicious node behavior is simulated here. An honest node will validate the block as is whereas a malicious node with the intent to overwrite the proposed block will broadcast a different block from the one it received. A malicious node with the intent to vote an invalid block is simulated as voting for a duplicate block that already exists in the chain. A node who wants to censor the proposer is marked by a flag censor_i. Prevote_1(), Prevote_2() and Prevote_3() share the same logical behavior with Prevote_0() and will be excluded from the code below.

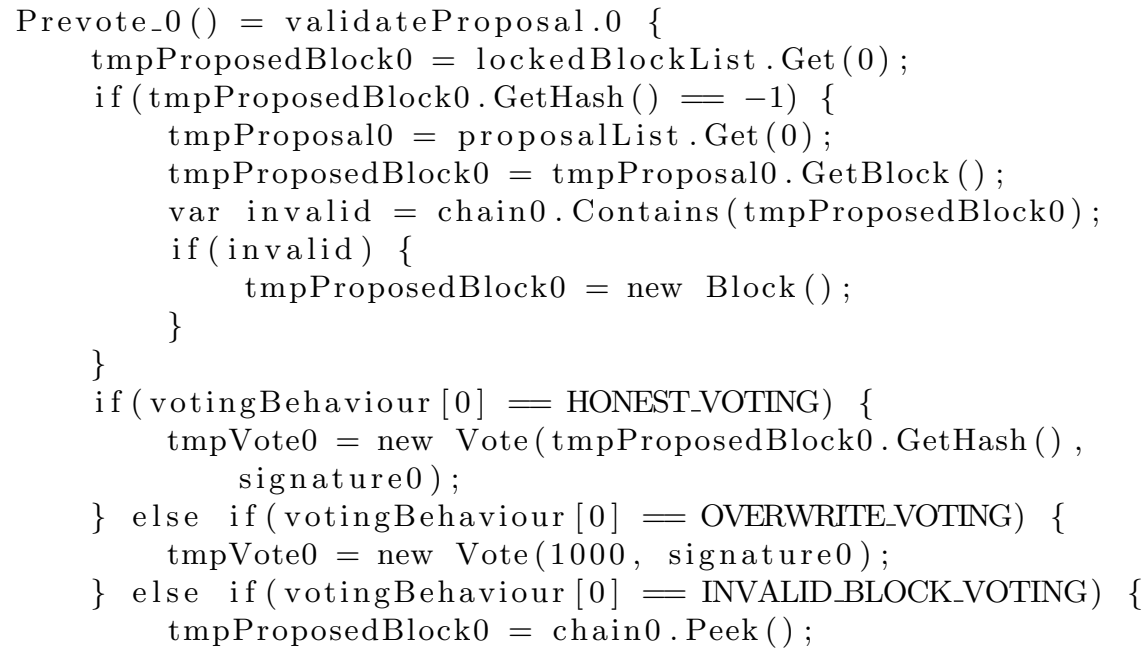




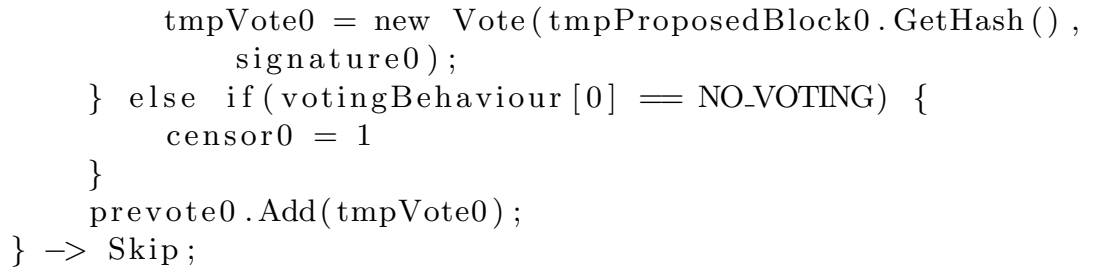

\subsection{Precommit}

The Precommit $(i)$ process is similar to Prevote $(i)$, defined by another sequence of two processes. The first process Precommit_i() will retrieve the first block with at least $67 \%$ confidence from the votes received from Prevote $(i)$ step. The second process of broadcasting and receiving is identical to that of Prevote $(i)$ and is excluded below.

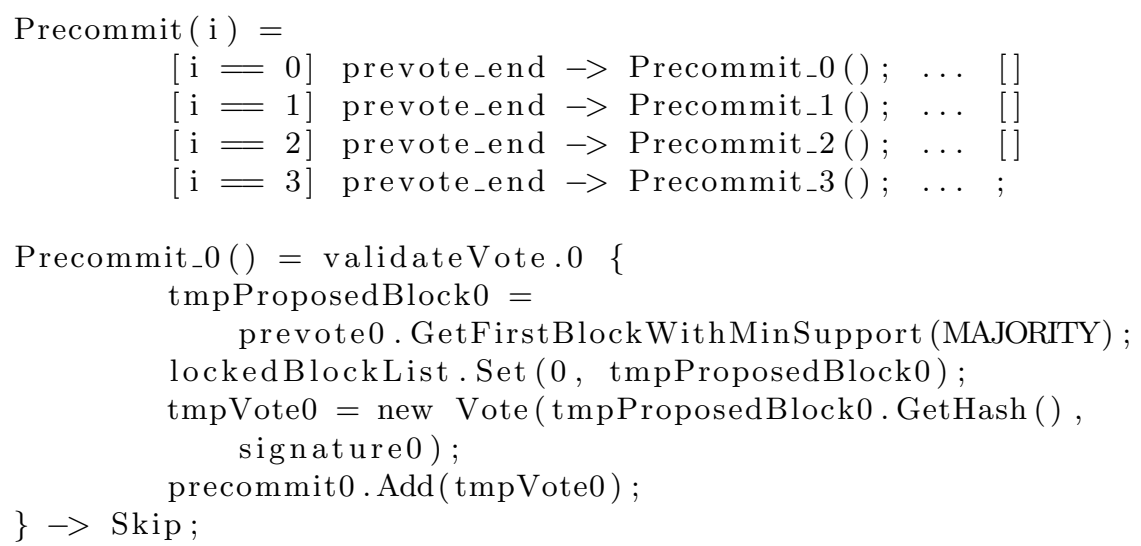

\subsection{Prepare Proof of Lock}

The PreparePOL $(i)$ process prepares the proof of lock, gathers the signatures of the validators who voted for this block and stores them inside the block.

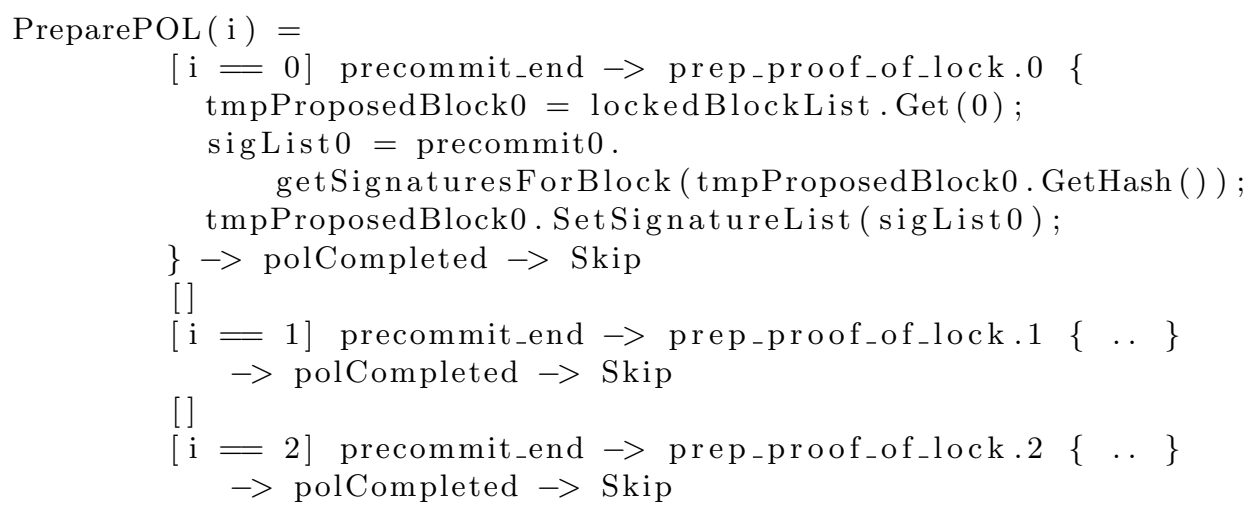




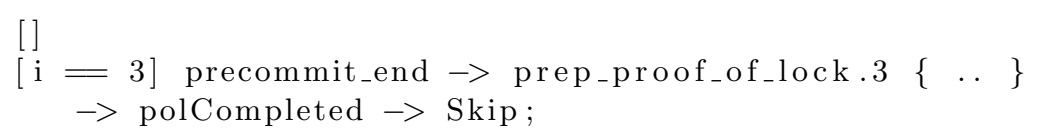

\subsection{Commit}

The Commit $(i)$ process adds the precommitted block having at least $67 \%$ consensus to the chain. The node will add the block to the chain only if it is not NIL. This marks the end of one round of consensus.

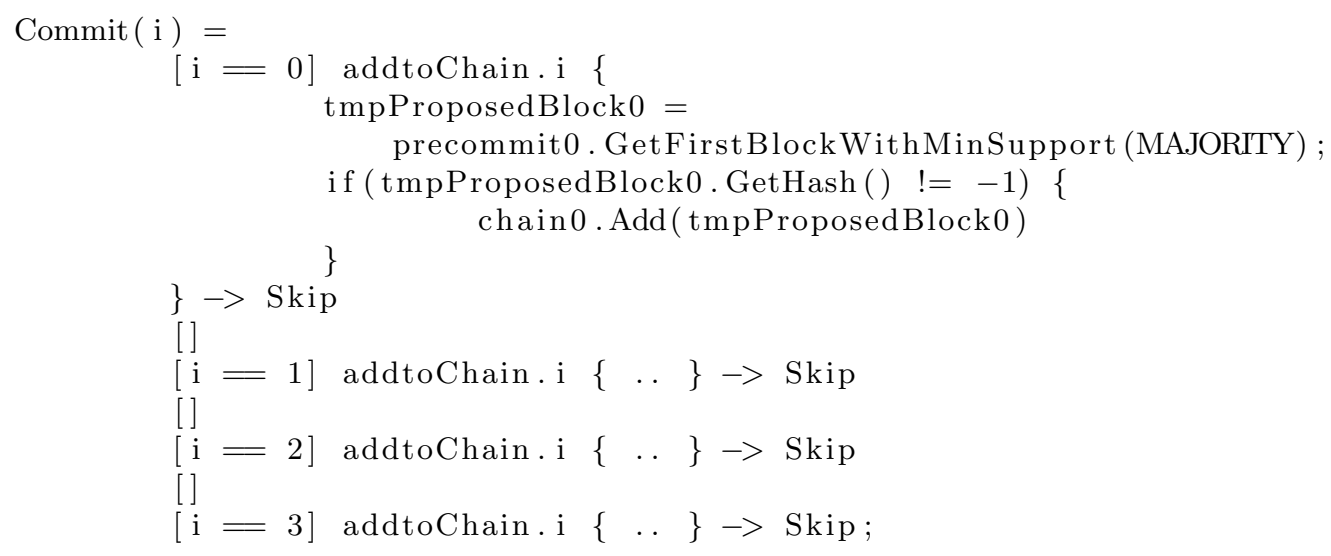

\section{Properties}

We will be verifying the properties of the model under normal conditions as well as against specific attacks in Byzantine environments. We define nine more variations of Blockchain () with different percentage of malicious nodes in the system with different threat factors.

BlockChainWithMinorityOverwrite () = Simulate Malicious (MINORITY, OVERWRITE_VOTING) ; BlockChain ();

BlockChainWithHalfOverwrite ()$=$ SimulateMalicious (HALF, OVERWRITE_VOTING); BlockChain ();

BlockChainWithMajorityOverwrite ()$=$ Simulate Malicious (MAJORITY, OVERWRITE_VOTING) ; BlockChain ();

BlockChainWithMinorityInvalid ()$=$ SimulateMalicious (MINORITY, INVALID_BLOCK_VOTING); BlockChain ( ) ;

BlockChainWithHalfInvalid ()$=$ SimulateMalicious (HALF, INVALID_BLOCK_VOTING); BlockChain ( ) ;

BlockChainWithMajorityInvalid () = SimulateMalicious (MAJORITY, INVALID_BLOCK_VOTING) ; BlockChain ( ) ;

BlockChainWithMinorityCensor ()$=$ SimulateMalicious (MINORITY, NO_VOTING); BlockChain () ; 
BlockChainWithHalfCensor ()$=$ SimulateMalicious (HALF, NO_VOTING); BlockChain ();

BlockChainWithMajorityCensor $($ ) = SimulateMalicious (MAJORITY, NO_VOTING); BlockChain ( ) ;

\subsection{Deadlockfree-ness}

A deadlock-free model means that at any point in time, no node should be waiting for another node to broadcast or receive a proposal or a vote, continue with validating of a block, or committing a block to its chain, even in Byzantine environments. We can use CSP \# to easily check for the deadlocks in the system as follows:

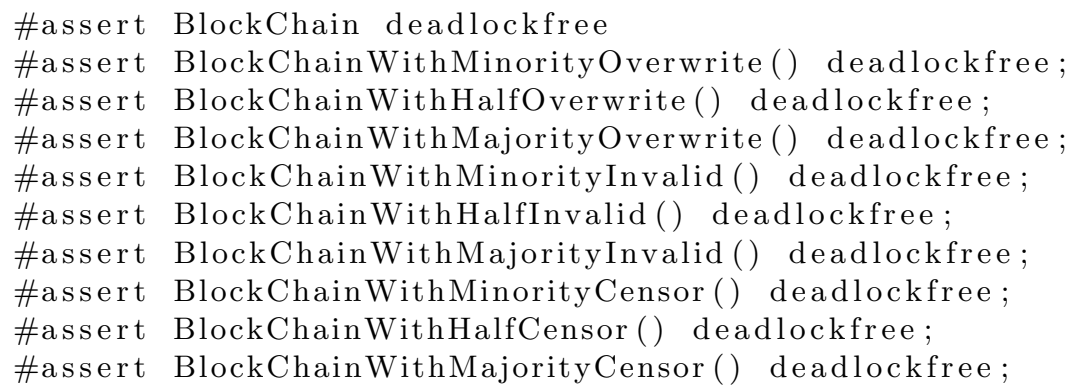

\subsection{Ability to reach consensus}

All the nodes in the network must be able to agree on one block and commit the same block to their own copy of the chain at the end of a round.

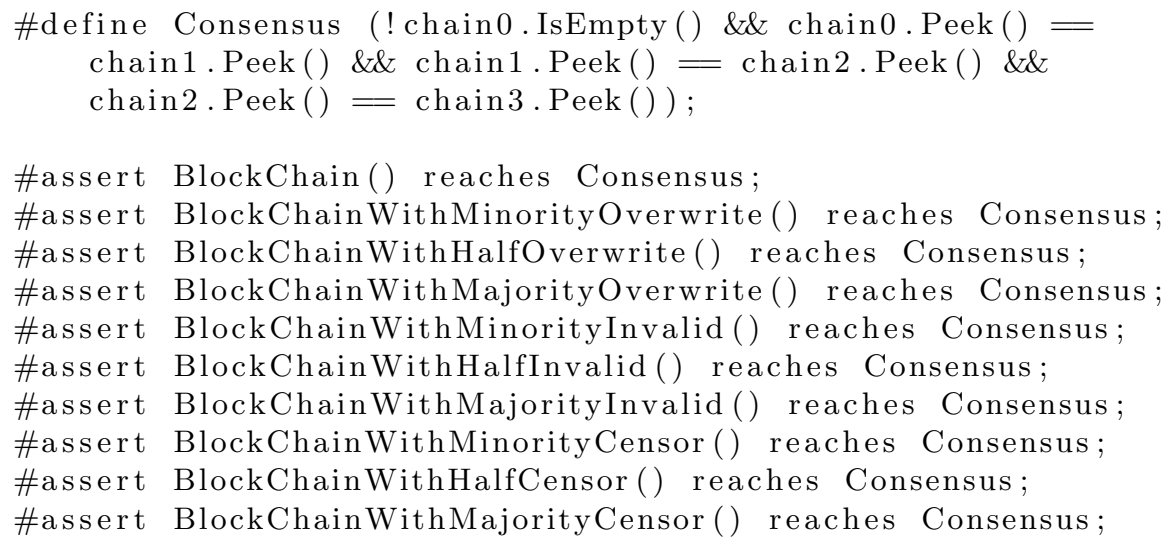

\subsection{Overwriting proposed block}

This is an attack where a set of nodes collude to reject the proposed block and propose a new block of their own. For a blockchain to be resistant to this attack, 
all nodes must agree on the same block to be added to the chain after reaching consensus and the block must be identical to the originally proposed block.

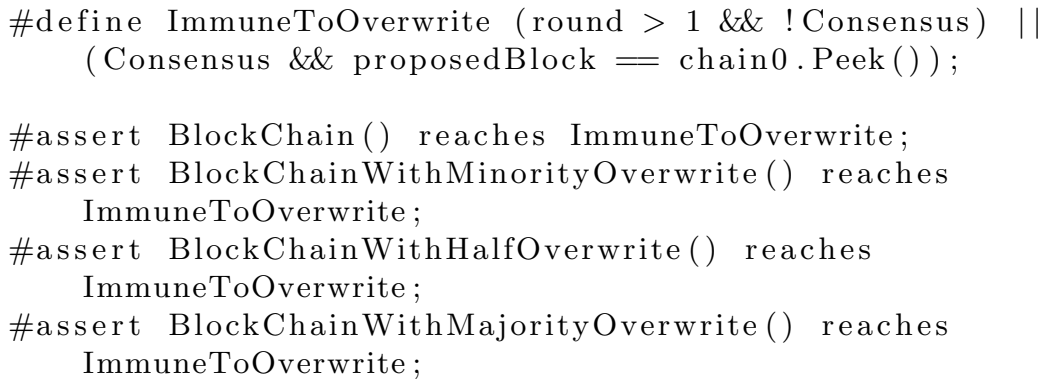

\subsection{Invalid blocks}

The network must be able to reject invalid blocks submitted by malicious nodes that do not conform to the rules of the blockchain. This is simulated by malicious nodes trying to add a duplicate (existing) block to the chain.

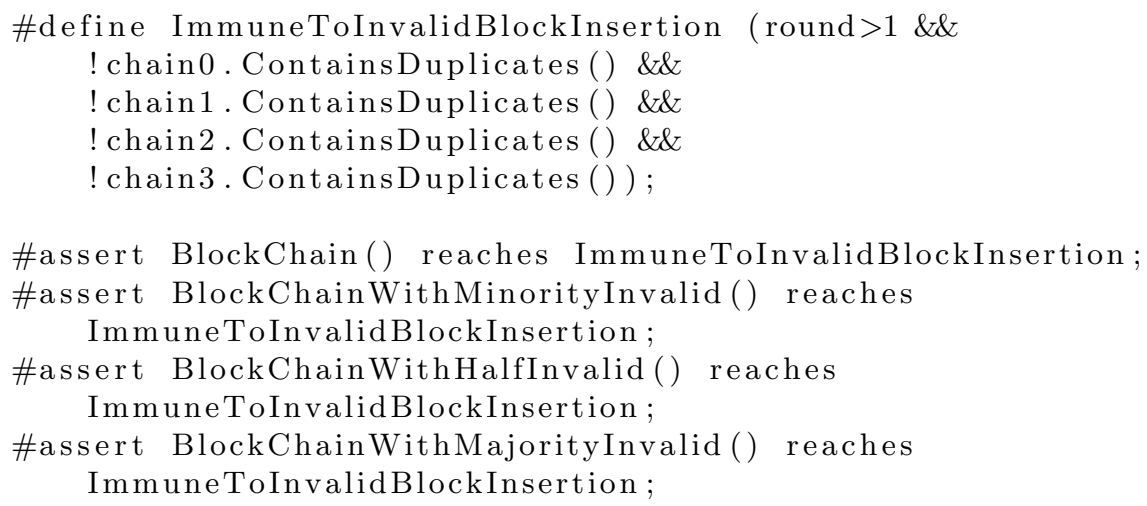

\subsection{Censorship attacks}

Censorship attack is an attack where malicious nodes in the network refuse to broadcast or vote a valid block in order to censor a particular content of the block or censor the node itself. The blockchain is immune to this attack if other nodes can reach consensus even with the absence of malicious nodes in the voting process. The assertions used to check for the reachability of consensus can be reuse for this verification.

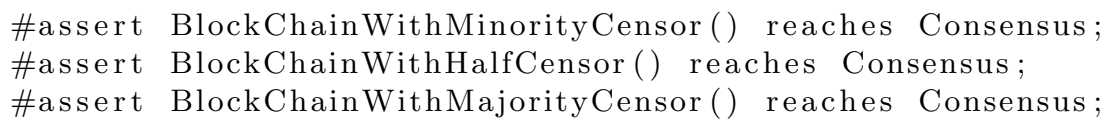




\section{Results and Analysis}

\subsection{General properties}

Table 1 presents the verification results of general properties of the model in normal and byzantine environments. The model is deadlock free at all times but does not always reach consensus. It is important to note that not being able to reach consensus does not always mean the system is vulnerable. Based on the threat model, it might be better for the network to agree on no block than to agree on the manipulated block. As such, consensus should be analyzed with respect to each individual threat model.

Table 1. Analysis results for deadlock-freeness and consensus reachability

\begin{tabular}{lcc}
\hline & Deadlock-free & Consensus \\
\hline BlockChain & $\checkmark$ & $\checkmark$ \\
BlockChain WithMinorityOverwrite $(\leqslant 33 \%)$ & $\checkmark$ & $\checkmark$ \\
BlockChainWithHalfOverwrite $(>33 \%$ and $<67 \%)$ & $\checkmark$ & $\boldsymbol{x}$ \\
BlockChainWithMajorityOverwrite $(\geqslant 67 \%)$ & $\checkmark$ & $\checkmark$ \\
BlockChainWithMinorityInvalid & $\checkmark$ & $\checkmark$ \\
BlockChainWithHalfInvalid & $\checkmark$ & $\boldsymbol{x}$ \\
BlockChainWithMajorityInvalid & $\checkmark$ & $\mathbf{x}$ \\
BlockChainWithMinorityCensor & $\checkmark$ & $\checkmark$ \\
BlockChainWithHalfCensor & $\checkmark$ & $\mathbf{x}$ \\
BlockChainWithMajorityCensor & $\checkmark$ & $\mathbf{x}$ \\
\hline
\end{tabular}

\subsection{Against proposed block overwrites}

From Table 2, we can see that BlockChain, BlockChainWithMinorityOverwrite and BlockChain WithHalfOverwrite are immune to overwrites. We have also learnt from Table 1 that BlockChain WithHalfOverwrite failed to reach consensus. By failing to reach consensus, the network did not commit a block during this round. This is in contrast to BlockChain WithMajorityOverwrite where the manipulated block voted by the majority $(\geqslant 67 \%)$ is added to the network instead of the proposed block.

Table 2. Analysis results for immunity to proposed block overwrite

\begin{tabular}{lc}
\hline & Immunity to block overwrite \\
\hline BlockChain & $\checkmark$ \\
BlockChain WithMinorityOverwrite $(\leqslant 33 \%)$ & $\checkmark$ \\
BlockChain WithHalfOverwrite $(>33 \%$ and $<67 \%)$ & $\checkmark$ \\
BlockChain WithMajorityOverwrite $(\geqslant 67 \%)$ & $\boldsymbol{x}$ \\
\hline
\end{tabular}

\subsection{Against invalid block insertions}

Here, we find that all four variations of the BlockChain can withstand the invalid block insertions. The only difference is that BlockChainWithMinorityInvalid 
and BlockChain are able to reach consensus whereas the other two are not. BlockChain WithMinorityInvalid is able to insert a valid block to the chain but no block is inserted to the chain in the case of the other two.

Table 3. Analysis results for immunity to invalid block insertions

\begin{tabular}{lc}
\hline & Immunity \\
\hline BlockChain & $\checkmark$ \\
BlockChain WithMinorityInvalid $(\leqslant 33 \%)$ & $\checkmark$ \\
BlockChain WithHalfInvalid $(>33 \%$ and $<67 \%)$ & $\checkmark$ \\
BlockChainWithMajorityInvalid $(\geqslant 67 \%)$ & $\checkmark$ \\
\hline
\end{tabular}

\subsection{Against censorship attacks}

As for the censorship attacks, we find that the protocol becomes vulnerable once the number of malicious nodes increases past $33 \%$. We have stated before that it takes $\geqslant 67 \%$ of the network to collude to vote on a same non-proposed block to add it to the blockchain. However, if the intent is not for overwriting but just for censorship or disruption of the service, this can be achieved with merely $>33 \%$ of the network just by having them not participating in the voting process. It is also possible that nodes get disconnected from the network without any malicious intent. In both cases, the chain will be prevented from adding new blocks until $\geqslant 67 \%$ of the network participates in the voting. Since the proposer is chosen by round robin, it is possible for the malicious minority nodes to stay offline until it is their turn to vote or until the next Propose step begins, effectively censoring the majority of the network from publishing anything.

Table 4. Analysis results for immunity to censorship

\begin{tabular}{lc}
\hline & Immunity to censorship attacks \\
\hline BlockChainWithMinorityCensor $(\leqslant 33 \%)$ & $\checkmark$ \\
BlockChainWithHalfCensor $(>33 \%$ and $<67 \%)$ & $\boldsymbol{x}$ \\
BlockChainWithMajorityCensor $(\geqslant 67 \%)$ & $\boldsymbol{x}$ \\
\hline
\end{tabular}

\section{Conclusions}

In this paper, we discussed the need for the formal analysis and verification of blockchain protocols. We implemented a simple consensus algorithm for a proof-of-stake blockchain based on Tendermint and verified that the algorithm is deadlock free. We also proved that it takes $\geqslant 67 \%$ of the network to reach consensus but it only takes $>33 \%$ of the network to censor the majority of the nodes from publishing a new block. It is possible to update the algorithm that chooses the proposer to ignore disconnected nodes in the previous round but this will only mitigate the problem. Alternative solutions to this are to implement a time lock consensus protocol [3] or to punish the nodes that are offline regardless 
of their intent, similar to how a node is punished by participating in malicious voting in proof-of-stake.

\section{References}

1. Bhargavan, K., Delignat-Lavaud, A., Fournet, C., Gollamudi, A., Gonthier, G., Kobeissi, N., Kulatova, N., Rastogi, A., Sibut-Pinote, T., Swamy, N., et al.: Formal verification of smart contracts: Short paper. In: Proceedings of the 2016 ACM Workshop on Programming Languages and Analysis for Security. pp. 91-96. ACM (2016)

2. Buchman, E.: Tendermint: Byzantine fault tolerance in the age of blockchains. Ph.D. thesis, The University of Guelph (2016)

3. Buterin, V.: The problem of censorship

(2015) https://blog.ethereum.org/2015/06/06/the-problem-of-censorship/

4. Buterin, V., Griffith, V.: Casper the friendly finality gadget. arXiv preprint arXiv:1710.09437 (2017)

5. Buterin, V., et al.: Ethereum white paper. GitHub repository (2013)

6. Eyal, I., Sirer, E.G.: Majority is not enough: Bitcoin mining is vulnerable. In: International conference on financial cryptography and data security. pp. 436-454. Springer (2014)

7. Garay, J., Kiayias, A., Leonardos, N.: The bitcoin backbone protocol: Analysis and applications. In: Annual International Conference on the Theory and Applications of Cryptographic Techniques. pp. 281-310. Springer (2015)

8. Kwon, J.: Tendermint: Consensus without mining. Retrieved May 18, 2017 (2014)

9. Mengelkamp, E., Notheisen, B., Beer, C., Dauer, D., Weinhardt, C.: A blockchainbased smart grid: towards sustainable local energy markets. Computer ScienceResearch and Development 33(1-2), 207-214 (2018)

10. P4Titan: Slimcoin: A peer-to-peer crypto-currency with proof-of-burn. GitHub repository (2014), https://github.com/slimcoin-project/slimcoinproject.github.io/raw/master/whitepaperSLM.pdf

11. Schwartz, D., Youngs, N., Britto, A., et al.: The ripple protocol consensus algorithm. Ripple Labs Inc White Paper 5 (2014)

12. Sharples, M., Domingue, J.: The blockchain and kudos: A distributed system for educational record, reputation and reward. In: European Conference on Technology Enhanced Learning. pp. 490-496. Springer (2016)

13. Sun, J., Liu, Y., Dong, J.S., Chen, C.: Integrating specification and programs for system modeling and verification. In: Theoretical Aspects of Software Engineering, 2009. TASE 2009. Third IEEE International Symposium on. pp. 127-135. IEEE (2009)

14. Sun, J., Liu, Y., Dong, J.S., Pang, J.: Pat: Towards flexible verification under fairness. In: International Conference on Computer Aided Verification. pp. 709714. Springer (2009)

15. Swan, M.: Blockchain: Blueprint for a new economy. " O’Reilly Media, Inc." (2015)

16. Zhang, Y., Wen, J.: An iot electric business model based on the protocol of bitcoin. In: Intelligence in Next Generation Networks (ICIN), 2015 18th International Conference on. pp. 184-191. IEEE (2015)

17. Zyskind, G., Nathan, O., et al.: Decentralizing privacy: Using blockchain to protect personal data. In: Security and Privacy Workshops (SPW), 2015 IEEE. pp. 180 184. IEEE (2015) 